OPEN ACCESS

Edited by: Milos Jesenak,

Comenius University, Slovakia

Reviewed by:

Wen-Chun Liu,

Academia Sinica, Taiwan

Jungang Chen,

University of Arkansas for Medical

Sciences, United States

${ }^{*}$ Correspondence:

Nima Rezaei

rezaei_nima@yahoo.com

${ }^{t}$ These authors have contributed equally to this work and

share first authorship

Specialty section:

This article was submitted to

Viral Immunology,

a section of the journal

Frontiers in Immunology

Received: 14 July 2021

Accepted: 28 September 2021

Published: 22 October 2021

Citation:

Karimi A, Shobeiri P, Kulasinghe $A$ and Rezaei N (2021) Novel Systemic Inflammation Markers to Predict COVID-19 Prognosis.

Front. Immunol. 12:741061. doi: 10.3389/fimmu.2021.741061

\section{Novel Systemic Inflammation Markers to Predict COVID-19 Prognosis}

\author{
Amirali Karimi ${ }^{1,2 \dagger}$, Parnian Shobeiri ${ }^{1,2,3,4 \dagger}$, Arutha Kulasinghe ${ }^{5}$ and Nima Rezaei ${ }^{2,3,6 *}$ \\ 1 School of Medicine, Tehran University of Medical Sciences, Tehran, Iran, ${ }^{2}$ Network of Immunity in Infection, Malignancy and \\ Autoimmunity (NIMA), Universal Scientific Education and Research Network (USERN), Tehran, Iran, ${ }^{3}$ Research Center for \\ Immunodeficiencies, Pediatrics Center of Excellence, Children's Medical Center, Tehran University of Medical Sciences, \\ Tehran, Iran, ${ }^{4}$ Non-Communicable Diseases Research Center, Endocrinology and Metabolism Population Sciences Institute, \\ Tehran University of Medical Sciences, Tehran, Iran, ${ }^{5}$ Centre for Genomics and Personalised Health, School of Biomedical \\ Q6 Sciences, Queensland University of Technology, Brisbane, QL, Australia, ${ }^{6}$ Department of Immunology, School of \\ Medicine, Tehran University of Medical Sciences, Tehran, Iran
}

Coronavirus disease 2019 (COVID-19) has resulted in a global pandemic, challenging both the medical and scientific community for the development of novel vaccines and a greater understanding of the effects of the SARS-CoV-2 virus. COVID-19 has been associated with a pronounced and out-of-control inflammatory response. Studies have sought to understand the effects of inflammatory response markers to prognosticate the disease. Herein, we aimed to review the evidence of 11 groups of systemic inflammatory markers for risk-stratifying patients and prognosticating outcomes related to COVID-19. Numerous studies have demonstrated the effectiveness of neutrophil to lymphocyte ratio (NLR) in prognosticating patient outcomes, including but not limited to severe disease, hospitalization, intensive care unit (ICU) admission, intubation, and death. A few markers outperformed NLR in predicting outcomes, including 1) systemic immune-inflammation index (SII), 2) prognostic nutritional index (PNI), 3) C-reactive protein (CRP) to albumin ratio (CAR) and high-sensitivity CAR (hsCAR), and 4) CRP to prealbumin ratio (CPAR) and highsensitivity CPAR (hSCPAR). However, there are a limited number of studies comparing NLR with these markers, and such conclusions require larger validation studies. Overall, the evidence suggests that most of the studied markers are able to predict COVID-19 prognosis, however NLR seems to be the most robust marker.

Keywords: biomarkers, COVID-19, inflammation, inflammatory markers, prognosis, SARS-CoV-2 


\section{INTRODUCTION}

Coronavirus disease 2019 (COVID-19) has emerged as a global challenge of the modern healthcare systems, resulting in more than 177 million confirmed cases and nearly 4 million deaths (1-3). Severe acute respiratory syndrome-coronavirus 2 (SARS-CoV-2) infection can involve various organs and produce a wide range of symptoms (4-9). Multiple organ involvement is thought to occur due to the almost universal distribution of angiotensinconverting enzyme 2 (ACE-2) that attaches to SARS-CoV-2 spike (S) protein receptor binding domain (RBD) and type 2 transmembrane serine proteases (TMPRSS2) that cleaves the $\mathrm{S}$ protein. It is thought that both these molecules may initiate immune evasion through various mechanisms (10-13).

It is well-documented that inflammatory mechanisms play a principal role in COVID-19-related organ dysfunction and mortality $(14,15)$. Patients with COVID-19 typically have higher inflammatory cytokines such as IL-6 and TNF- $\alpha$ compared with healthy individuals (16). Furthermore, patients with COVID-19 experience elevated levels of serologic indicators of inflammation, such as C-reactive protein (CRP), erythrocyte sedimentation rate (ESR), lactate dehydrogenase (LDH), and procalcitonin $(17,18)$. These inflammatory cytokines may also alter the levels of various blood cell lineages and notably cause lymphocytopenia $(18,19)$. This hyperinflammation plays an important role in viral pathogenesis. However, it is also possible to use this proinflammatory response to risk-stratify COVID-19 patients at high risk of developing severe disease and respiratory complications (20).

Historically, markers of inflammation were used to successfully prognosticate patients with inflammatory diseases and, in particular, various types of cancers (21-25). Previous studies examined the role of inflammatory markers in other infectious diseases and demonstrated their ability to risk-stratify patients (26). Herein, we aimed to review the evidence for the effectiveness of systemic inflammatory markers in riskstratifying patients and prognosticating outcomes related to COVID-19. The markers include neutrophil to lymphocyte ratio (NLR) and derived NLR (d-NLR), platelet to lymphocyte ratio (PLR), lymphocyte to monocyte ratio (LMR), lymphocyte to CRP ratio (LCR), fibrinogen to prealbumin ratio (FPR) and albumin to fibrinogen ratio (AFR), CRP to albumin ratio (CAR) and CRP to prealbumin ratio (CPAR), Glasgow prognostic score (GPS), modified GPS (mGPS), high-sensitivity mGPS (HSmGPS), prognostic index (PI), prognostic nutritional index (PNI), systemic immune-inflammation index (SII), and interferon-alpha-inducible protein 27 (IFI27).

\section{INFLAMMATORY MARKERS}

\subsection{Neutrophil to Lymphocyte Ratio and Derived NLR}

NLR is defined as the absolute neutrophil count (ANC)/absolute lymphocyte count (ALC) (27-29). d-NLR has a similar definition to NLR, calculated as ANC/(White blood cells (WBC) total count
- ANC) (30). If we consider monocyte, basophil, and eosinophil levels as negligible (which are mostly not), the definition of these two markers would be equal. Earlier studies found links to higher NLR or d-NLR in chronic conditions with low-grade inflammatory nature, such as obesity, hypertension, diabetes mellitus, metabolic syndrome, atherosclerotic events of the heart and brain, and various cancers, although previous literature studied NLR more than d-NLR (27-29, 31-33). These underlying diseases are considered as risk factors for severe COVID-19 (2, 34, 35).

Since the beginning of the pandemic, studies have investigated the role of NLR in COVID-19 prognostication and its utility as a biomarker. NLR has been reported to prognosticate mortality, progression to severe disease, risk of intubation, risk of severe disease in intubated patients, days intubated, ICU admission, and longer intensive care unit (ICU) admission (30, 36-57). Two meta-analyses of $n=19$ and $n=13$ studies found significant associations between higher NLR and COVID-19 severity and mortality $(58,59)$. Furthermore, patients with higher NLR appear to have more comorbidities and, therefore, are more prone to severe COVID-19 (36). Even in patients with comorbidities, NLR might maintain its predictive ability for COVID-19 severity. For instance, NLR significantly predicted COVID-19 severity and survival in hospitalized patients with different types of cancers $(60,61)$. It has been suggested that each increased NLR unit resulted in an $8 \%$ higher mortality in COVID-19 patients (45).

A temporal analysis showed that on-admission NLR correlates well with the need for ICU and poor outcomes, and can be a potential risk-stratification tool. However, the clinical utility of NLR was lost in week 3 post-admission (62). The best predictive value of NLR can be achieved at its peak compared with its on-admission values (63). Concurrently, another study on the temporality of NLR found that day 7 measurement of NLR could significantly predict those requiring invasive mechanical ventilation and mortality, while measurement of day 1 NLR could not (64). In summary, on-admission NLR could predict COVID-19 prognosis. This predictive ability increases for a few days after admission, when NLR reaches its peak. However, NLR gradually loses its predictive ability as the patient recovers from COVID-19 and an associated reduction in inflammation. Finally, at week 3 post-admission, NLR loses its clinical utility to prognosticate severe COVID-19 outcomes.

Five studies proposed the ability of NLR to assist COVID-19 diagnosis $(49,65-68)$. They defined assisting COVID-19 diagnosis as significantly higher levels of NLR in individuals with COVID-19 compared with healthy controls. However, none of the studies mentioned how and due to what situations NLR can be integrated into COVID-19 diagnosis. Two other markers have been reported to be predictive for COVID-19 disease severity and mortality: granulocyte to lymphocyte ratio (69) and d-NLR $(30,70,71)$.

\subsubsection{NLR in Comparison With Other Markers}

In Tables 1, 2, we compared NLR to other reported markers for COVID-19. We seperated the variables into those that have been reported for COVID-19 diagnosis and disease severity. 
TABLE 1 | Studies comparing NLR to only PLR, LMR, and d-NLR among different measured variables.

\begin{tabular}{|c|c|c|}
\hline Study & Measured variable & Summary of findings \\
\hline (49) & COVID-19 diagnosis & In intubated COVID-19 patients: higher NLR and PLR and lower LMR were observed compared with healthy individuals. \\
\hline (65) & COVID-19 diagnosis & NLR, PLR, and MLR were all higher in COVID-19 patients [order of higher AUC: MLR (0.892) > PLR (0.748) > NLR (0.722)]. \\
\hline (66) & COVID-19 diagnosis & Both NLR and PLR correlated (order of higher correlation: NLR > PLR). \\
\hline (67) & COVID-19 diagnosis & Both NLR and PLR were higher is SARS-CoV-2 (+) patients [order of higher AUC: PLR (0.669) > NLR (0.615)]. \\
\hline (30) & COVID-19 pneumonia & NLR correlated in the multivariate analysis, but d-NLR, LMR, and PLR did not. \\
\hline (30) & Disease severity & NLR, d-NLR, and PLR correlated with disease severity (order of better prediction: NLR $>$ d-NLR $>$ PLR). LMR did not correlate. \\
\hline (59) & Disease severity & In this meta-analysis, NLR correlated better than PLR (SMD: 2.80 vs. 1.82). \\
\hline$(72)$ & Disease severity & $\begin{array}{l}\text { In patients with type } 2 \text { diabetes mellitus: both NLR and LMR correlated with disease severity (AUC: NLR }=0.730, p=0.002 \text {; } \\
\text { LMR }=0.322, p=0.015 \text { ). }\end{array}$ \\
\hline (73) & Disease severity & $\begin{array}{l}\text { NLR, d-NLR, and PLR correlated with disease severity [order of higher AUC: NLR }(0.808)>\text { d-NLR }(0.803)>\text { PLR }(0.769)] \text {. LMR did } \\
\text { not correlate (AUC = 0.296). }\end{array}$ \\
\hline (74) & Disease severity & $\begin{array}{l}\text { NLR remained independently related in the logistic regression analysis. PLR only correlated in the univariate analysis. No correlation } \\
\text { was observed for LMR. }\end{array}$ \\
\hline (53) & Disease severity & NLR, PLR, and LMR could predict disease severity (order of higher AUC: NLR > LMR > PLR). \\
\hline (65) & Progression to ARDS & NLR, PLR, and LMR predicted progression to ARDS. \\
\hline (53) & ICU admission & Among patients with severe disease, NLR correlated with ICU admission, but LMR and PLR did not. \\
\hline (75) & ICU admission & NLR, PLR, and LMR predicted ICU admission (order of better prediction: NLR > PLR > LMR). \\
\hline (76) & Mechanical ventilation & NLR predicted the need for mechanical ventilation, but PLR did not. \\
\hline (72) & Time to negative PCR & In patients with type 2 diabetes mellitus, NLR correlated (multivariate analysis), but LMR did not (univariate analysis). \\
\hline (72) & Duration of hospital stay & In patients with type 2 diabetes mellitus, NLR independently correlated but LMR was not related in the univariate analysis. \\
\hline$(77)$ & In-hospital mortality & NLR and d-NLR correlated, but LMR and PLR did not. \\
\hline (66) & Mortality & NLR correlated, but PLR did not. \\
\hline (78) & All-cause mortality & NLR could predict this parameter, but PLR could not. \\
\hline$(72)$ & Hospital expenses & In patients with type 2 diabetes mellitus, NLR independently correlated but LMR did not correlate in the univariate analysis. \\
\hline
\end{tabular}

$N L R$, neutrophil to lymphocyte ratio; PLR, platelet to lymphocyte ratio; LMR, lymphocyte to monocyte ratio; MLR, monocyte to lymphocyte ratio; $d-N L R$, derived-NLR; AUC, area under the curve; SMD, standardized mean difference; $A R D S$, acute respiratory distress syndrome; ICU, intensive care unit; PCR, polymerase chain reaction.

TABLE 2 | Studies comparing NLR with other biomarkers (studies involving discussed markers other than PLR, LMR, and d-NLR) among different measured variables.

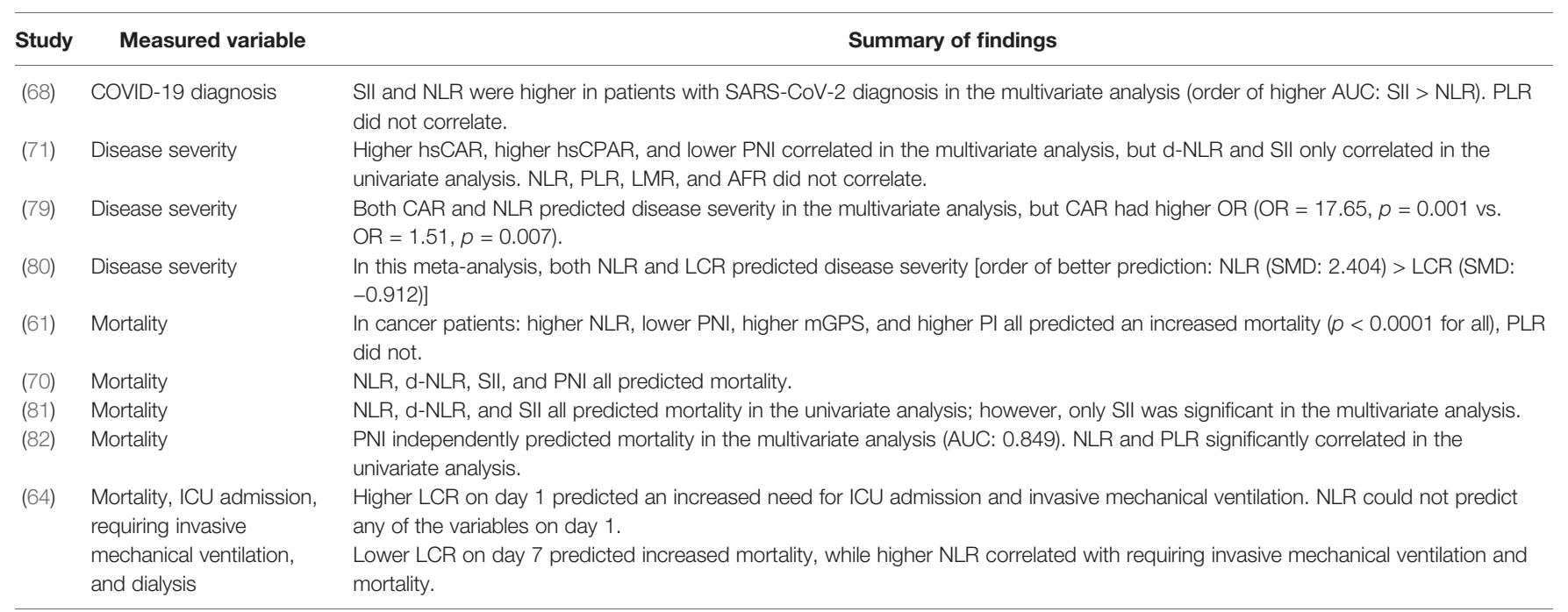

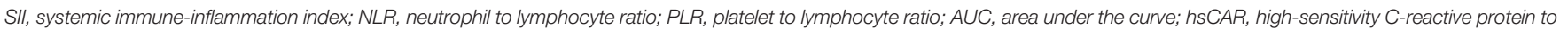

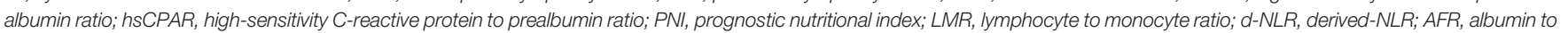

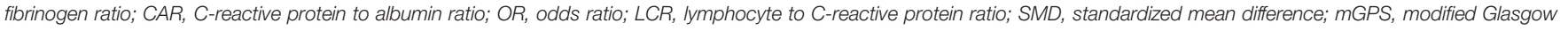
prognostic score; $\mathrm{Pl}$, prognostic index.

Table 1 summarizes the studies comparing NLR to only LMR, PLR, and d-NLR. NLR had the highest predictive value compared with LMR, PLR, and d-NLR in most of the studies for severe COVID-19 parameters-disease severity, ICU admission, progression to acute respiratory distress syndrome (ARDS), need for mechanical ventilation, duration and expense of hospital stay, time to negative PCR, and mortality.
We first compared disease severity reported by seven studies (Table 1) $(30,53,59,71-74)$. One of these was a meta-analysis of 20 studies, 19 on NLR and 5 on PLR, that found a correlation between higher NLR and PLR with disease severity. However, the mean standardized difference (SMD) for NLR was higher than PLR (2.80 versus 1.82) (59). Five of the six remaining studies found NLR superior to d-NLR, PLR, and $\operatorname{LMR}(30,53,71-74)$. 
The other study found d-NLR to be the only predictive marker in the univariate but not multivariate analysis among these four. NLR, PLR, and LMR did not correlate with disease severity (71).

NLR, PLR, and LMR could predict ICU admission in hospitalized patients; however, NLR (AUC: 0.861) could predict ICU admission better than PLR (AUC: 0.715) and LMR (AUC: 0.705) (75). Sun et al. concluded similarly and stated that only NLR correlated with the risk of ICU admission, while LMR and PLR did not (53). NLR, monocyte to lymphocyte ratio (MLR), and PLR could all predict progression to ARDS (65). Higher NLR could predict the need for mechanical ventilation ( $p=0.003$ ), but PLR was similar between patients requiring ventilation and those not $(p=0.41)(76)$.

NLR outperformed in prognosticating mortality compared with PLR $(61,66,77,78)$ and $\operatorname{LMR}(77)$. Three studies comparing NLR and d-NLR found that both could predict mortality (70, $77,81)$.

While NLR had a greater predictive power for severe COVID19 parameters, it did not seem to correlate with COVID-19 diagnosis compared with PLR and LMR. The studies defined correlating with COVID-19 diagnosis as having significantly different levels in COVID-19 positive and negative patients. Five studies compared NLR, PLR, and LMR based on their diagnostic ability (Table 1) (49, 65-68). Lissoni et al. specifically compared intubated COVID-19 patients and healthy individuals. They concluded that lower LMR, higher NLR, and higher PLR were observed in intubated patients with COVID-19 compared with healthy controls (49). Among the remaining four studies, NLR correlated better than PLR in two studies $(66,68)$ and worse in two others $(65,67)$. Only one of these studies contained the MLR, the inverted LMR variable (65). In this study, MLR had the highest AUC to differentiate healthy individuals from COVID-19 patients (0.892), followed by PLR (0.748) and NLR (0.722) (65). Overall, NLR was not superior to LMR and PLR in assisting diagnosis, but data are insufficient on this part to determine the best marker.

Only nine studies compared NLR to markers other than PLR, LMR, and d-NLR (61, 64, 68, 70, 71, 79-82) (Table 2). These studies provide valuable evidence but are not sufficient for an extensive assessment. Five of these studies measured NLR and SII $(61,68,70,71,81)$, two of them without the possibility to compare the predictive ability of NLR and SII $(61,70)$. These two studies-one of them in cancer patientsfound that NLR, d-NLR, SII, PNI, and mGPS could predict COVID-19 mortality, but it was not possible to determine the best predictive marker in these studies $(61,70)$. SII was superior to NLR in all the other three remaining studies comparing NLR and SII $(68,71,81)$; one study related COVID-19 diagnosis (68), one for disease severity (71), and one for mortality (81). SII was also superior to d-NLR, MLR, and PLR in predicting mortality, with a small hazard ratio $(\mathrm{HR}=1.0001, p=0.029)(81)$.

Xue et al. concluded that hsCAR, hsCPAR, and PNI predicted COVID-19 severity in the multivariate analysis, while d-NLR and SII only correlated in the univariate analysis. NLR, LMR, PLR, and AFR could not predict severe COVID-19 (71).
This study concluded the superiority of hsCAR, hsCPAR, and PNI. PNI was superior to NLR in predicting mortality and CAR in predicting disease severity, each in one study $(79,82)$.

In the study of Lagunas-Rangel, NLR was superior to LCR in predicting disease severity (SMD: NLR $=2.404, \mathrm{LCR}=-0.912$ ), although both were significant predictors (P: NLR $=0.001$, LCR < 0.001) (80).

Altogether, these data suggest that some markers might produce more promising results than NLR, such as SII, PNI, CAR and hsCAR, and CPAR and hsCPAR. However, these markers are less studied compared with NLR. Although this section contained some of the comparison of other variables, a detailed discussion on each marker follows.

\subsection{Platelet to Lymphocyte Ratio}

PLR could help in diagnosing COVID-19. Four studies found a significant difference in the PLR of patients with positive SARSCoV-2 compared to healthy individuals $(49,65-67)$, while only one concluded against this (68).

Two meta-analyses confirmed the effectiveness of higher PLR on predicting COVID-19 severity $(59,83)$. Higher PLR also correlated with an increased risk of severe disease in intubated COVID-19 patients (49). This ability to predict disease severity seemed to be optimal at its peak. PLR at peak could predict disease severity in the multivariate regression analysis; however, PLR at admission did not correlate with disease severity in the univariate analysis (84). Two studies studied the ability of PLR to predict ICU admission, and they produced conflicting results on this matter $(53,75)$.

Although PLR could predict disease severity in most of the studies, it was not able to predict mortality $(61,66,77,78)$, one specifically in cancer patients (61). Similarly, another study concluded that PLR is only slightly prognostic in predicting mortality in the univariate analysis among hospitalized patients $(p<0.001)$, but not in the multivariate analysis $(p=0.154)(82)$.

Owing to all the strengths of PLR in predicting various COVID-19-related parameters, it is a potentially suitable marker to triage COVID-19 patients. However, it seems to lack potentials to predict mortality and have a lower ability than NLR to predict several parameters.

\subsection{Lymphocyte to Monocyte Ratio}

Unlike neutrophil and monocyte count, a decrease in lymphocyte count correlated to multiorgan injury in COVID19 patients (85). This was shown by Kazancioglu et al recently. However, in their study, monocyte count only correlated to SARS-CoV-2 infection but not severity (69). These studies provide the hypothetical bases for the prognostic value of LMR in COVID-19, as well as NLR and PLR.

Two studies compared the effectiveness of LMR in COVID-19 diagnosis, both finding a significant relationship between LMR and testing positive for SARS-CoV-2. In one of them, MLR (AUC: 0.892 ) was the best predictor compared with NLR and PLR, and 0.23 was declared the best MLR cutoff point (65). In another, significantly lower LMR was observed in intubated COVID-19 patients compared with healthy controls (49). 
LMR did not correlate with disease severity in most studies $(30,71,73,74)$ except two $(53,72)$. Liu et al. showed that higher LMR could only significantly predict disease severity in the univariate analysis and also did not correlate with a longer hospital stay, higher hospital costs, and longer time to negative PCR (72). LMR could prognosticate progression to ARDS (65).

In two studies examining the ability to predict ICU admission, LMR did not correlate in one (53), and correlated but was inferior to NLR and PLR in the other (75). Data are limited regarding the ability of LMR to predict COVID-19 mortality; however, a study concluded the ineffectiveness of LMR in prognosticating this parameter (77).

LMR might have limited benefits in prognosticating COVID19 (86), but its abilities seem to be lower than NLR and PLR, especially in predicting disease severity, ICU admission, and mortality. However, we encourage future studies to pursue the ability of LMR to recognize SARS-CoV-2 positive patients, as it demonstrated promises.

\subsection{Lymphocyte to C-Reactive Protein Ratio}

A limited number of studies examined this marker. The most important article on this is perhaps a meta-analysis on the role of LCR in predicting disease severity. They found a significantly lower LCR in patients with severe disease (SMD $=-0.912$, $p<0.001)$; however, it was less predictive compared with NLR (SMD $=2.404, p=0.001)$. They based their results on five studies for each marker (80).

Higher LCR on day 1 predicted the need for ICU admission (adjusted OR: 3.1, $p=0.003$ ) and invasive mechanical ventilation (adjusted OR: 2.5, $p=0.009$ ), but could not predict in-hospital mortality $(p=0.60)$ and requiring dialysis $(p=0.44)$. Nevertheless, lower LCR on day 7 only correlated with an increased in-hospital mortality risk (adjusted OR: 0.1, 95\% $\mathrm{CI}=0.01-0.30, p<0.0001)$ but not with the other factors (64).

\subsection{Fibrinogen to Prealbumin Ratio and Albumin to Fibrinogen Ratio}

Similar to most of the discussed markers, studies demonstrate a prognostic role for FPR and AFR in some cancers and other diseases with inflammatory pathophysiology (87-89). Nevertheless, only a few articles studied them to determine COVID-19 disease severity.

Lower AFR correlated with severe COVID-19 in univariate analysis $(p<0.0001)$, but not multivariate analysis $(p=0.079)$ (71). However, fibrinogen to albumin ratio (FAR), remained significant in predicting disease severity in the multivariate analysis in another study $(\mathrm{HR}=4.058,95 \% \mathrm{CI}=1.246-13.222$, $p=0.020)(90)$.

\subsection{C-Reactive Protein to Albumin Ratio and C-Reactive Protein to Prealbumin Ratio}

CAR could predict disease severity in two studies $(79,91)$ : one comparing it with NLR and finding a higher OR for CAR $(\mathrm{OR}=17.652, p=0.001)$ than NLR $(\mathrm{OR}=1.512, p=0.007)(79)$.
However, the other study did not find as large an OR for CAR (1.264, $p=0.037$ ) (91).

hsCAR and hsCPAR differ from their counterparts as they utilize high-sensitivity CRP (92). Xue et al. found that onadmission hsCAR, hsCPAR, and PNI significantly correlated with severe COVID-19 in the multivariate analysis among several other markers (NLR, LMR, FPR, PLR, SII, AFR). Furthermore, among these markers, only hsCPAR and hsCAR correlated with hospital stay length (71). In the Oh et al. study, hsCAR could also predict in-hospital mortality in adults older than 65 years of age after adjusting for confounders (92).

Taken together, CAR and CPAR seemed promising in predicting disease severity, mortality, and length of hospital stay in all the studies, although we only identified four studies. Furthermore, a small study hailed prealbumin and CRP as potential markers to effectively triage patients in the early stages, and prealbumin seemed to be more effective (93).

\subsection{Glasgow Prognostic Score, Modified GPS, and High-Sensitivity mGPS}

GPS constitutes two main serum components, CRP and albumin levels, both having a potential of 0 or 1 score. CRP $>10 \mathrm{mg} / \mathrm{L}$ and albumin $<3.5 \mathrm{mg} / \mathrm{dl}$ receive one point each, and the score classifies the patients into three total scores of 0,1 , or 2 . mGPS does not allocate a score to hypoalbuminemia without a rise in CRP to above $10 \mathrm{mg} / \mathrm{L}$. hs-mGPS provides a similar classification to mGPS with a lower CRP threshold (>3 mg/L) $(94,95)$. These markers also have proven roles in predicting various cancers $(94,95)$.

In a study of 397 patients with COVID-19, no deaths occurred among 40 patients with hs-mGPS score of 0 , while $10 / 263(3.80 \%)$ and $24 / 94(25.53 \%)$ of patients scoring 1 and 2 died, respectively (70). Concurrently, Dettorre et al. found that mGPS was able to foretell the overall survival of hospitalized cancer patients infected with COVID-19 (11.4\%, 30.4\%, and $50.6 \%$ for $\mathrm{mGPS}=0$ to $\mathrm{mGPS}=2$, respectively; $p<0.0001)(61)$. These two studies found promising results for mGPS and hsmGPS and set the grounds for future research to better identify its effectiveness.

\subsection{Prognostic Index}

$\mathrm{PI}$ is similar to the GPS, only differing in the WBC component. $\mathrm{WBC}>11,000 / \mu \mathrm{l}$ and $\mathrm{CRP}>10 \mathrm{mg} / \mathrm{L}$ contribute to this scoring system of 0 to 2 (22).

We could only find one study discussing this marker in COVID-19. In that study, PI was able to predict the survival of cancer patients infected with COVID-19 (9.1\%, 40\%, and 50\%, for scores of 0 to 2), similar to NLR, PNI, and mGPs. PI seemed superior to PLR in the study. Patients categorized in the poorrisk group $(\mathrm{PI}=2)$ had 23 days median overall survival, while patients with favorable scores did not reach the required followup duration (all $p$-values less than 0.0001) (61).

\subsection{Prognostic Nutritional Index}

Onodera et al. proposed PNI as an immune-nutritional risk score for malnourished cancer patients undergoing for gastrointestinal 
surgery, formulizing it as serum albumin concentration $(\mathrm{g} / \mathrm{L})+$ $0.005 \times$ total lymphocyte count (per $\mathrm{mm}^{3}$ of peripheral blood) $(96,97)$. This marker later demonstrated its effectiveness in prognosticating several types of cancer (98).

Three studies investigated the relationship between COVID19 mortality and PNI, all showing significant correlations (61, 70, 82). Two studies were on hospitalized COVID-19 patients (70, 82 ) and another on patients with cancer (61). PNI also predicted disease severity better than other markers in the study of Xue et al., alongside hsCAR, and hsCPAR. Together, they were put into a nomogram that could predict disease severity well $(\mathrm{C}$-index $=0.873)(71)$.

PNI successfully predicted disease severity and mortality in all the four studies examining it; therefore, it can be a suitable candidate for follow-up studies.

\subsection{Systemic Immune-Inflammation Index}

SII is defined as platelet count $\times$ NLR (99). SII remained a reliable predictor in most of the conducted studies on COVID-19 so far. Usul et al. found its superior predictive ability than NLR and PLR in COVID-19 diagnosis, as its values were significantly different in SARS-CoV-2-positive and -negative individuals. The proposed SII for helping in the COVID-19 diagnosis was 479.1 (68).

Xue et al. studied the relationship between several markers and disease severity. They found that SII could significantly predict disease severity in the univariate but not the multivariate analysis, inferior to hsCAR, hsCPAR, and PNI, but still better than several markers, such as NLR (71).

Two studies studied SII in predicting mortality, both finding significant correlations $(70,81)$. One of them found that SII was the only significant marker in the multivariate analysis, superior to NLR and d-NLR, but with a slight hazard ratio $(\mathrm{HR}=1.0001, p=0.029)(81)$.

\subsection{Interferon-Alpha Inducible Protein 27}

IFI27 is a part of the innate immune system highly induced by interferon (IFN)- $\alpha$ (100). High expression of IFI27 may also induce cell proliferation and invasion and reduce apoptosis, making it a possible oncogene $(100,102)$.

Type I IFN deficiency can be a marker of severe COVID-19 (103). Some of the IFN-stimulated genes (ISGs) like IFI27 were upregulated in patients with COVID-19 (104-107) and later downregulated in the recovery process $(108,109)$. IFI27 was overexpressed in various cell lineages of SARS-CoV-2-infected patients compared with healthy controls (110). In another study, IFI27 demonstrated a higher than two-fold upregulation in A549 and normal human bronchial epithelial (NHBE) cells infected with SARS-CoV-2 (111). Shaath et al. analyzed the bronchoalveolar lavage of 10 individuals. IFI27 was among the genes upregulated in the peripheral blood mononuclear cells (PBMC) of severe and mild COVID-19 patients, compared with two healthy controls (112).

IFI27 might also help distinguish COVID-19 from other acute respiratory illnesses and some viral diseases such as
Ebola, SARS, MERS, and H1N1, as SARS-CoV-2, even at low loads, induced IFI27 more than other viruses $(113,114)$.

There is a lack of robust clinical evidence concerning IFI27related prognostic value for COVID-19; however, ISGs and, particularly, IFI27 seem to be interesting for conducting further studies.

\section{CONCLUSION}

NLR seems to have the highest prognosticating potential among the biomarkers discussed in this study, because of its predictive value and availability for data across multiple studies, including meta-analyses. Therefore, this amplitude of evidence might increase its reliability to risk-stratify patients and help medical decision-making. Nevertheless, some other markers might also be promising, such as SII, PNI, CAR and hsCAR, and CPAR and hsCPAR, but other aspects of their prognostication need to be further studied (Figure 1). Careful comparisons require future meta-analyses.

Several studies discussed the markers for specific subgroups, such as patients with underlying diabetes or cancer. Many of these conditions might be inflammatory in nature, and they might hypothetically alter the effectiveness of some markers.

The need for risk-stratifying COVID-19 patients also encouraged some researchers to design new markers for this purpose that should be examined in studies, such as COVID-19 severity-Iraqi index (CSI) measured by MLR $\times$ lactate dehydrogenase (LDH)/upper normal LDH value (115). Another study hypothesized combining functional and nutritional indices with the well-known CURB-65 pneumonia severity index (116).

Some of the presented markers may only require a complete blood count with differentials, a cheap and straightforward test. The other markers also require routine and widely available laboratory tests. Therefore, stratifying the risks of patients using these methods has the potential of being widely available.

Some important pitfalls and limitations exist that future research need to address. First, studies need to estimate the cost-effectiveness of triaging the patients with these biomarkers, as almost all of them seemed to be useful to various degrees. Second, there is a lack of sufficient evidence for many of these biomarkers. Some of these markers have the potential to be better prognosticators than NLR, but need further studies to confirm their abilities and provide sufficient evidence. Third, we encourage researchers to hypothesize novel biomarkers bestfitted to COVID-19 pathophysiology and test their hypotheses to understand their effectiveness. We also encourage future research on specific subgroups with certain underlying conditions, as the most suitable biomakers for those groups might differ from the overall population. At last, various COVID-19 variants are showing different specific outcomes of morbidity and mortality (117). Therefore, we suggest future researchers to update the findings related to systemic inflammatory markers specifically for emerging variants. 


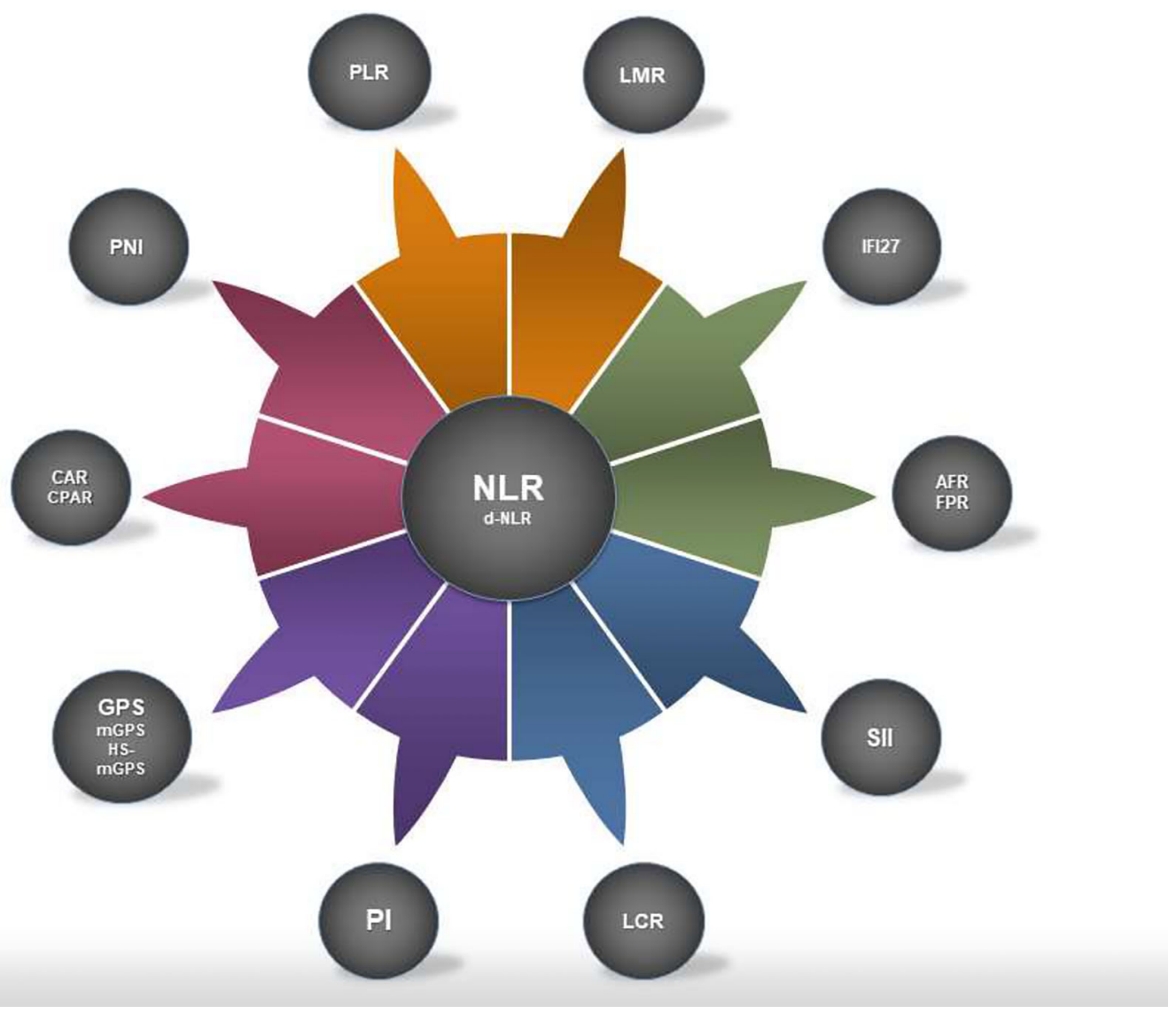

FIGURE 1 | Systemic inflammation markers discussed in this study. NLR is illustrated in the middle as it was the most studied marker with strong prognosticating capabilities. However, some of the less studied markers reviewed in the study might have the potential to overtake the predictive value of NLR. NLR, neutrophil to lymphocyte ratio; d-NLR, derived NLR; PLR, platelet to lymphocyte ratio; LMR, lymphocyte to monocyte ratio; LCR, lymphocyte to C-reactive protein ratio; FPR, fibrinogen to prealbumin ratio; AFR, albumin to fibrinogen ratio; CAR, C-reactive protein to albumin ratio; CPAR, C-reactive protein to prealbumin ratio; GPS, Glasgow prognostic score; mGPS, modified GPS; hs-mGPS, high-sensitivity mGPS; PI, prognostic index; PNI, prognostic nutritional index; SIl, systemic immuneinflammation index; IFI27, interferon-alpha inducible protein 27.

\section{AUTHOR CONTRIBUTIONS}

AKa: conception, drafting of the initial manuscript, and revision of the final manuscript. PS: conception, drafting of the initial

\section{REFERENCES}

1. Lambert H, Gupte J, Fletcher H, Hammond L, Lowe N, Pelling M, et al. COVID-19 as a Global Challenge: Towards an Inclusive and Sustainable Future. Lancet Planet Health (2020) 4(8):e312-e4. doi: 10.1016/S2542-5196 (20)30168-6

2. Mehraeen E, Karimi A, Barzegary A, Vahedi F, Afsahi AM, Dadras O, et al. Predictors of Mortality in Patients With COVID-19-a Systematic Review. Eur J Integr Med (2020) 40:101226. doi: 10.1016/j.eujim.2020.101226

3. The World Health Organization. WHO Coronavirus Disease (COVID-19) Dashboard (2021). Available at: https://covid19.who.int/.

4. Shafi AMA, Shaikh SA, Shirke MM, Iddawela S, Harky A. Cardiac Manifestations in COVID-19 Patients-A Systematic Review. J Cardiac Surg (2020) 35(8):1988-2008. doi: 10.1111/jocs.14808 manuscript, and figure visualization. AKu: conception and careful revision of the manuscript draft. NR: conception and careful revision of the manuscript draft. All authors contributed to the article and approved the submitted version.

5. Mehraeen E, Seyed Alinaghi SA, Nowroozi A, Dadras O, Alilou S, Shobeiri P, et al. A Systematic Review of ECG Findings in Patients With COVID-19. Indian Heart J (2020) 72(6):500-7. doi: 10.1016/j.ihj.2020.11.007

6. Qiu Y, He J-S, Tan J-Y, Lin S-N, Mao R. Gastrointestinal and Liver Involvement in Patients With COVID-19 - Authors' Reply. Lancet Gastroenterol Hepatol (2020) 5(9):799-800. doi: 10.1016/S2468-1253(20) 30240-5

7. Thaweerat W. Current Evidence on Pancreatic Involvement in SARS-CoV-2 Infection. Pancreatology (2020) 20(5):1013-4. doi: 10.1016/j.pan.2020. 05.015

8. Momtazmanesh S, Shobeiri P, Hanaei S, Mahmoud-Elsayed H, Dalvi B, Rad EM. Cardiovascular Disease in COVID-19: A Systematic Review and MetaAnalysis of 10,898 Patients and Proposal of a Triage Risk Stratification Tool. Egypt Heart J (2020) 72(1):1-17. doi: 10.1186/s43044-020-00075-Z 
9. Seyed ASA, Karimi A, Shobeiri P, Nowroozi A, Mehraeen E, Afsahi AM, et al. Psychological Symptoms of COVID-19 Epidemic: A Systematic Review of Current Evidence. Psihologija (2020) 54(2)173-92. doi: 10.2298/ PSI200703035S

10. SeyedAlinaghi S, Mehrtak M, MohsseniPour M, Mirzapour P, Barzegary A, Habibi P, et al. Genetic Susceptibility of COVID-19: A Systematic Review of Current Evidence. Eur J Med Res (2021) 26(1):46. doi: 10.1186/s40001-02100516-8

11. Shang J, Wan Y, Luo C, Ye G, Geng Q, Auerbach A, et al. Cell Entry Mechanisms of SARS-CoV-2. Proc Natl Acad Sci (2020) 117(21):11727. doi: 10.1073/pnas.2003138117

12. Mollica V, Rizzo A, Massari F. The Pivotal Role of TMPRSS2 in Coronavirus Disease 2019 and Prostate Cancer. Future Oncol (London England) (2020) 16 (27):2029-33. doi: 10.2217/fon-2020-0571

13. Deng Q, Rasool RU, Russell RM, Natesan R, Asangani IA. Targeting Androgen Regulation of TMPRSS2 and ACE2 as a Therapeutic Strategy to Combat COVID-19. iScience (2021) 24(3):102254. doi: 10.1016/ j.isci.2021.102254

14. Merad M, Martin JC. Pathological Inflammation in Patients With COVID19: A Key Role for Monocytes and Macrophages. Nat Rev Immunol (2020) 20(6):355-62. doi: 10.1038/s41577-020-0331-4

15. Vabret N, Samstein R, Fernandez N, Merad M. Advancing Scientific Knowledge in Times of Pandemics. Nat Rev Immunol (2020) 20(6):338. doi: 10.1038/s41577-020-0319-0

16. Del Valle DM, Kim-Schulze S, Huang H-H, Beckmann ND, Nirenberg S, Wang B, et al. An Inflammatory Cytokine Signature Predicts COVID-19 Severity and Survival. Nat Med (2020) 26(10):1636-43. doi: 10.1038/s41591020-1051-9

17. Ali N. Elevated Level of C-Reactive Protein May Be an Early Marker to Predict Risk for Severity of COVID-19. J Med Virol (2020) 92(11):2409-11. doi: $10.1002 / j m v .26097$

18. Ghahramani S, Tabrizi R, Lankarani KB, Kashani SMA, Rezaei S, Zeidi N, et al. Laboratory Features of Severe vs.Non-Severe COVID-19 Patients Asian Populations: syst Rev meta-anal. Eur J Med Res (2020) 25(1):30. doi: 10.1186/s40001-020-00432-3

19. Tavakolpour S, Rakhshandehroo T, Wei EX, Rashidian M. Lymphopenia During the COVID-19 Infection: What It Shows and What Can Be Learned. Immunol Lett (2020) 225:31-2. doi: 10.1016/j.imlet.2020.06.013

20. RECOVERY Collaborative Group, Horby P, Lim WS, Emberson JR, Mafham M, Bell JL, et al. Dexamethasone in Hospitalized Patients With Covid-19. New Engl J Med (2020) 384(8):693-704. doi: 10.1056/ NEJMoa2021436

21. Wang C, He W, Yuan Y, Zhang Y, Li K, Zou R, et al. Comparison of the Prognostic Value of Inflammation-Based Scores in Early Recurrent Hepatocellular Carcinoma After Hepatectomy. Liver Int Off J Int Assoc Study Liver (2020) 40(1):229-39. doi: 10.1111/liv.14281

22. Yamamoto M, Kobayashi T, Kuroda S, Hamaoka M, Okimoto S, Honmyo N, et al. Verification of Inflammation-Based Prognostic Marker as a Prognostic Indicator in Hepatocellular Carcinoma. Ann gastroenterol Surg (2019) 3 (6):667-75. doi: 10.1002/ags3.12286

23. Cui K, Ou Y, Shen Y, Li S, Sun Z. Clinical Value of Circulating Tumor Cells for the Diagnosis and Prognosis of Hepatocellular Carcinoma (HCC): A Systematic Review and Meta-Analysis. Medicine (2020) 99(40):e22242. doi: 10.1097/MD.0000000000022242

24. Tang M, Jia Z, Zhang J. The Prognostic Role of Prognostic Nutritional Index in Nasopharyngeal Carcinoma: A Systematic Review and MetaAnalysis. Int J Clin Oncol (2021) 26(1):66-77. doi: 10.1007/s10147-02001791-x

25. Sirin MC, Korkmaz S, Erturan I, Filiz B, Aridogan BC, Cetin ES, et al. Evaluation of Monocyte to HDL Cholesterol Ratio and Other Inflammatory Markers in Patients With Psoriasis. Anais brasileiros dermatol (2020) 95 (5):575-82. doi: 10.1016/j.abd.2020.02.008

26. Russell CD, Parajuli A, Gale HJ, Bulteel NS, Schuetz P, de Jager CPC, et al. The Utility of Peripheral Blood Leucocyte Ratios as Biomarkers in Infectious Diseases: A Systematic Review and Meta-Analysis. J Infect (2019) 78(5):33948. doi: 10.1016/j.jinf.2019.02.006

27. Templeton AJ, McNamara MG, Šeruga B, Vera-Badillo FE, Aneja P, Ocaña A, et al. Prognostic Role of Neutrophil-to-Lymphocyte Ratio in Solid
Tumors: A Systematic Review and Meta-Analysis. JNCI: J National Cancer Institute (2014) 106:(6):dju124. doi: 10.1093/jnci/dju124

28. Tokgoz S, Kayrak M, Akpinar Z, Seyithanoğlu A, Güney F, Yürüten B, et al. Neutrophil Lymphocyte Ratio as a Predictor of Stroke. J Stroke Cerebrovascular Dis (2013) 22(7):1169-74. doi: 10.1016/j.jstrokecerebrovasdis.2013.01.011

29. Balta S, Celik T, Mikhailidis DP, Ozturk C, Demirkol S, Aparci M, et al. The Relation Between Atherosclerosis and the Neutrophil-Lymphocyte Ratio. Clin Appl Thrombosis/Hemostasis (2016) 22(5):405-11. doi: 10.1177/ 1076029615569568

30. Yang A-P, Liu J-P, Tao W-Q, Li H-M. The Diagnostic and Predictive Role of NLR, D-NLR and PLR in COVID-19 Patients. Int Immunopharmacol (2020) 84:106504. doi: 10.1016/j.intimp.2020.106504

31. Karaman M, Balta S, Ahmet AYS, Cakar M, Naharci I, Demirkol S, et al. The Comparative Effects of Valsartan and Amlodipine on vWf Levels and N/L Ratio in Patients With Newly Diagnosed Hypertension. Clin Exp Hypertens (2013) 35(7):516-22. doi: 10.3109/10641963.2012.758734

32. Balta S, Kurtoglu E, Kucuk U, Demirkol S, Ozturk C. Neutrophillymphocyte Ratio as an Important Assessment Tool. Expert Rev Cardiovascular Ther (2014) 12: (5):537-8. doi: 10.1586/14779072. 2014.902309

33. Caputo D, Caricato M, Coppola A, La Vaccara V, Fiore M, Coppola R. Neutrophil to Lymphocyte Ratio (NLR) and Derived Neutrophil to Lymphocyte Ratio (D-NLR) Predict Non-Responders and Postoperative Complications in Patients Undergoing Radical Surgery After Neo-Adjuvant Radio-Chemotherapy for Rectal Adenocarcinoma. Cancer Invest (2016) 34 (9):440-51. doi: 10.1080/07357907.2016.1229332

34. Zheng Z, Peng F, Xu B, Zhao J, Liu H, Peng J, et al. Risk Factors of Critical \& Mortal COVID-19 Cases: A Systematic Literature Review and MetaAnalysis. J Infect (2020) 81(2):e16-25. doi: 10.1016/j.jinf.2020.04.021

35. Meng Y, Lu W, Guo E, Liu J, Yang B, Wu P, et al. Cancer History is an Independent Risk Factor for Mortality in Hospitalized COVID-19 Patients: A Propensity Score-Matched Analysis. J Hematol Oncol (2020) 13(1):75. doi: 10.1186/s13045-020-00907-0

36. Tatum D, Taghavi S, Houghton A, Stover J, Toraih E, Duchesne JJS Neutrophil-To-Lymphocyte Ratio and Outcomes in Louisiana Covid-19 Patients. Shock (2020) 54(5):652-8. doi: 10.1097/SHK.0000000000001585

37. Cheng B, Hu J, Zuo X, Chen J, Li X, Chen Y, et al. Predictors of Progression From Moderate to Severe Coronavirus Disease 2019: A Retrospective Cohort. Clin Microbiol Infect (2020) 26(10):1400-5. doi: 10.1016/ j.cmi.2020.06.033

38. Yan X, Li F, Wang X, Yan J, Zhu F, Tang S, et al. Neutrophil to Lymphocyte Ratio as Prognostic and Predictive Factor in Patients With Coronavirus Disease 2019: A Retrospective Cross-Sectional Study. J Medical Virol (2020) 92(11):2573-81. doi: 10.1002/jmv.26061

39. Asghar MS, Kazmi SJH, Khan NA, Akram M, Khan SA, Rasheed U, et al. Clinical Profiles, Characteristics, and Outcomes of the First 100 Admitted COVID-19 Patients in Pakistan: A Single-Center Retrospective Study in a Tertiary Care Hospital of Karachi. Cureus (2020) 12(6):e8712. doi: 10.7759/ cureus.c34

40. Basbus L, Lapidus MI, Martingano I, Puga MC, Pollán JJM. Neutrophil to Lymphocyte Ratio as a Prognostic Marker in COVID-19. Medicina (Buenos Aires) (2020) 80:31-6.

41. Li H, Zhao M, Xu Y. Biochemical Analysis Between Common Type and Critical Type of COVID-19 and Clinical Value of Neutrophil/Lymphocyte Ratio. Nan Fang Yi Ke Da Xue Xue Bao (2020) 40(7):965-71. doi: 10.12122/ j.issn.1673-4254.2020.07.08

42. Ok F, Erdogan O, Durmus E, Carkci S, Canik A. Predictive Values of Blood Urea Nitrogen/Creatinine Ratio and Other Routine Blood Parameters on Disease Severity and Survival of COVID-19 Patients. J Medical Virol (2020) 93(2):786-93. doi: 10.1002/jmv.26300

43. Zhang B, Zhou X, Zhu C, Song Y, Feng F, Qiu Y, et al. Immune Phenotyping Based on the Neutrophil-to-Lymphocyte Ratio and IgG Level Predicts Disease Severity and Outcome for Patients With COVID-19. Front Mol Biosci (2020) 7:157. doi: 10.3389/fmolb.2020.00157

44. Zhang J-J, Cao Y-Y, Tan G, Dong X, Wang B-C, Lin J, et al. Clinical, Radiological, and Laboratory Characteristics and Risk Factors for Severity and Mortality of 289 Hospitalized COVID-19 Patients. Allergy (2020) 76 (2):533-50. doi: 10.22541/au.159164076.65659447 
45. Liu Y, Du X, Chen J, Jin Y, Peng L, Wang HH, et al. Neutrophil-ToLymphocyte Ratio as an Independent Risk Factor for Mortality in Hospitalized Patients With COVID-19. J Infect (2020) 81(1):e6-12. doi: 10.1016/j.jinf.2020.04.002

46. Liu Y-P, Li G-M, He J, Liu Y, Li M, Zhang R, et al. Combined Use of the Neutrophil-to-Lymphocyte Ratio and CRP to Predict 7-Day Disease Severity in 84 Hospitalized Patients With COVID-19 Pneumonia: A Retrospective Cohort Study. Ann Transl Med (2020) 8(10):635. doi: 10.21037/atm-20-2372

47. Xia X, Wen M, Zhan S, He J, Chen W. [An Increased Neutrophil/ Lymphocyte Ratio Is an Early Warning Signal of Severe COVID-19]. Nan fang yi ke da xue xue bao = J South Med Univ (2020) 40(3):333-6. doi: 10.12122/j.issn.1673-4254.2020.03.06

48. Vafadar Moradi E, Teimouri A, Rezaee R, Morovatdar N, Foroughian M, Layegh P, et al. Increased Age, Neutrophil-to-Lymphocyte Ratio (NLR) and White Blood Cells Count Are Associated With Higher COVID-19 Mortality. Am J Emergency Med (2021) 40:11-4. doi: 10.1016/j.ajem.2020.12.003

49. Lissoni P, Rovelli F, Monzon A, Privitera C, Messina G, Porro GJJIA. Evidence of Abnormally Low Lymphocyte-to-Monocyte Ratio in Covid-19Induced Severe Acute Respiratory Syndrome. J Immunol Allergy (2020) 1 (2):1-6. doi: 10.37191/Mapsci-2582-6549-1(2)-011

50. Fei M, Tong F, Tao X, Wang J. Value of Neutrophil-to-Lymphocyte Ratio in the Classification Diagnosis of Coronavirus Disease 2019. Zhonghua Wei Zhong Bing Ji Jiu Yi Xue (2020) 32(5):554-8. doi: 10.3760/cma.j.cn12143020200413-00506

51. Liu J, Liu Y, Xiang P, Pu L, Xiong H, Li C, et al. Neutrophil-To-Lymphocyte Ratio Predicts Critical Illness Patients With 2019 Coronavirus Disease in the Early Stage. J Transl Med (2020) 18:1-12. doi: 10.1186/s12967-020-02374-0

52. Shang W, Dong J, Ren Y, Tian M, Li W, Hu J, et al. The Value of Clinical Parameters in Predicting the Severity of COVID-19. J Medical Virol (2020) 92(10):2188-92. doi: 10.1002/jmv.26031

53. Sun S, Cai X, Wang H, He G, Lin Y, Lu B, et al. Abnormalities of Peripheral Blood System in Patients With COVID-19 in Wenzhou, China. Clin Chimica Acta (2020) 507:174-80. doi: 10.1016/j.cca.2020.04.024

54. Wang C, Deng R, Gou L, Fu Z, Zhang X, Shao F, et al. Preliminary Study to Identify Severe From Moderate Cases of COVID-19 Using Combined Hematology Parameters. Ann Transl Med (2020) 8(9):593. doi: 10.21037/ atm-20-3391

55. Zha Q, Feng B, Li X, Zhou D, Kang Y, Qin HJLM. Study on Early Laboratory Warning of Severe COVID-19. Lab Med (2020) 35(06):557-60.

56. Chen F-F, Zhong M, Liu Y, Zhang Y, Zhang K, Su D-Z, et al. The Characteristics and Outcomes of 681 Severe Cases With COVID-19 in China. J Crit Care (2020) 60:32-7. doi: 10.1016/j.jcrc.2020.07.003

57. Song H, Bai T, Shi J, Yang J. Predictive Value of Multiple Inflammatory Indexes on the Prognosis of Patients With Corona Virus Disease 2019. Pract J Cardiac Cereb Pneumal Vascular Dis (2020) 28(06):13-6.

58. Li X, Liu C, Mao Z, Xiao M, Wang L, Qi S, et al. Predictive Values of Neutrophil-to-Lymphocyte Ratio on Disease Severity and Mortality in COVID-19 Patients: A Systematic Review and Meta-Analysis. Crit Care (2020) 24(1):647. doi: 10.1186/s13054-020-03374-8

59. Chan AS, Rout A. Use of Neutrophil-To-Lymphocyte and Platelet-ToLymphocyte Ratios in COVID-19. J Clin Med Res (2020) 12(7):448-53. doi: $10.14740 /$ jocmr 4240

60. Ma J, Yin J, Qian Y, Wu Y. Clinical Characteristics and Prognosis in Cancer Patients With COVID-19: A Single Center's Retrospective Study. J Infect (2020) 81(2):318-56. doi: 10.1016/j.jinf.2020.04.006

61. Dettorre G, Diamantis N, Loizidou A, Piccart M, Chester J, Jackson A, et al. 319o The Systemic Pro-Inflammatory Response Identifies Cancer Patients With Adverse Outcomes From SARS-CoV-2 Infection. Ann Oncol (2020) 31:S1366-S. doi: 10.1016/j.annonc.2020.10.313

62. Khourssaji M, Chapelle V, Evenepoel A, Belkhir L, Yombi JC, van Dievoet M-A, et al. A Biological Profile for Diagnosis and Outcome of COVID-19 Patients. Clin Chem Lab Med (CCLM) (2020) 58(12):2141-50. doi: 10.1515/ cclm-2020-0626

63. Jimeno S, Ventura PS, Castellano JM, García-Adasme SI, Miranda M, Touza $\mathrm{P}$, et al. Prognostic Implications of Neutrophil-Lymphocyte Ratio in COVID-19. Eur J Clin Invest (2021) 51(1):e13404. doi: 10.1111/eci.13404
64. Ullah W, Basyal B, Tariq S, Almas T, Saeed R, Roomi S, et al. LymphocyteTo-C-Reactive Protein Ratio: A Novel Predictor of Adverse Outcomes in COVID-19. J Clin Med Res (2020) 12(7):415-22. doi: 10.14740/jocmr4227

65. Peng J, Qi D, Yuan G, Deng X, Mei Y, Feng L, et al. Diagnostic Value of Peripheral Hematologic Markers for Coronavirus Disease 2019 (COVID19): A Multicenter, Cross-Sectional Study. J Clin Lab Anal (2020) 34(10): e23475. doi: 10.1002/jcla.23475

66. Eslamijouybari M, Heydari K, Maleki I, Moosazadeh M, HedayatizadehOmran A, Vahedi L, et al. Neutrophil-To-Lymphocyte and Platelet-ToLymphocyte Ratios in COVID-19 Patients and Control Group and Relationship With Disease Prognosis. Caspian J Intern Med (2020) 11 (Suppl 1):531-5. doi: 10.22088/cjim.11.0.531

67. Seyit M, Avci E, Nar R, Senol H, Yilmaz A, Ozen M, et al. Neutrophil to Lymphocyte Ratio, Lymphocyte to Monocyte Ratio and Platelet to Lymphocyte Ratio to Predict the Severity of COVID-19. Am J Emergency Med (2021) 40:110-4. doi: 10.1016/j.ajem.2020.11.058

68. Usul E, Şan İ, Bekgöz B, Şahin A. Role of Hematological Parameters in COVID-19 Patients in the Emergency Room. Biomarkers Med (2020) 14 (13):1207-15. doi: 10.2217/bmm-2020-0317

69. Kazancioglu S, Yilmaz FM, Bastug A, Sakallı A, Ozbay BO, Buyuktarakci C, et al. Lymphocyte Subset Alteration and Monocyte CD4 Expression Reduction in Patients With Severe COVID-19. Viral Immunol (2020) 34 (5):342-51. doi: 10.1089/vim.2020.0166

70. Doganci S, Ince M, Ors N, Yildirim A, Sir E, Karabacak K, et al. A New COVID-19 Prediction Scoring Model for in-Hospital Mortality: Experiences From Turkey, Single Center Retrospective Cohort Analysis. Eur Rev Med Pharmacol Sci (2020) 24(19):10247-57. doi: 10.26355/eurrev_202010_23249

71. Xue G, Gan X, Wu Z, Xie D, Xiong Y, Hua L, et al. Novel Serological Biomarkers for Inflammation in Predicting Disease Severity in Patients With COVID-19. International Immunopharmacology. Int Immunopharmacol (2020) 89(Pt A):107065. doi: 10.1016/j.intimp.2020.107065

72. Liu G, Zhang S, Hu H, Liu T, Huang J. The Role of Neutrophil-Lymphocyte Ratio and Lymphocyte-Monocyte Ratio in the Prognosis of Type 2 Diabetics With COVID-19. Scottish Med J (2020) 65(4):154-60. doi: 10.1177/ 0036933020953516

73. Noor A, Akhtar F, Tashfeen S, Anwar N, Saleem B, Khan SA, et al. Neutrophil-To-Lymphocyte Ratio, Derived Neutrophil-to-Lymphocyte Ratio, Platelet-to-Lymphocyte Ratio and Lymphocyte-to-Monocyte Ratio as Risk Factors in Critically Ill Covid-19 Patients, a Single Centered Study. J Ayub Med Coll Abbottabad (2020) 32(4-sup):S595-601.

74. Huang S, Huang M, Li X, Zhang T, Lu H. Significance of Neutrophil-toLymphocyte Ratio, Platelet-to-Lymphocyte Ratio for Predicting Clinical Outcomes in COVID-19. Medrxiv (2020). doi: 10.1101/2020.05.04.20090431

75. Bastug A, Bodur H, Erdogan S, Gokcinar D, Kazancioglu S, Kosovali BD, et al. Clinical and Laboratory Features of COVID-19: Predictors of Severe Prognosis. Int Immunopharmacol (2020) 88:106950. doi: 10.1016/ j.intimp.2020.106950

76. Nair PR, Maitra S, Ray BR, Anand RK, Baidya DK, Subramaniam R. Neutrophil-To-Lymphocyte Ratio and Platelet-To-Lymphocyte Ratio as Predictors of the Early Requirement of Mechanical Ventilation in COVID-19 Patients. Indian J Crit Care Med (2020) 24(11):1143-4. doi: 10.5005/jp-journals-10071-23663

77. Panagiotis P, Angelo Z, Valentina S, Giulia M, Giacomo De R, Rosa MP, et al. Laboratory Test Alterations in Patients With COVID-19 and non COVID-19 Interstitial Pneumonia: A Preliminary Report. J Infect Developing Countries (2020) 14(07):685-90. doi: 10.3855/jidc.12879

78. Wang X, Li X, Shang Y, Wang J, Zhang X, Su D, et al. Ratios of Neutrophilto-Lymphocyte and Platelet-to-Lymphocyte Predict All-Cause Mortality in Inpatients With Coronavirus Disease 2019 (COVID-19): A Retrospective Cohort Study in a Single Medical Centre. Epidemiol Infect (2020) 148:e211. doi: $10.1017 / S 0950268820002071$

79. Wang $\mathrm{X}, \mathrm{Xu} \mathrm{Y}$, Huang $\mathrm{H}$, Jiang $\mathrm{D}$, Zhou $\mathrm{C}$, Liao $\mathrm{H}$, et al. Increased Pretreatment C-Reactive Protein-to-Albumin Ratio Predicts Severe Coronavirus Disease 2019. Researchsquare (2020). doi: 10.21203/rs.3.rs$31723 / \mathrm{v} 2$

80. Lagunas-Rangel FA. Neutrophil-To-Lymphocyte Ratio and Lymphocyte-toC-Reactive Protein Ratio in Patients With Severe Coronavirus Disease 2019 
(COVID-19): A Meta-Analysis. J Med Virol (2020) 92(10):1733-4. doi: 10.1002/jmv. 25819

81. Fois AG, Paliogiannis P, Scano V, Cau S, Babudieri S, Perra R, et al. The Systemic Inflammation Index on Admission Predicts In-Hospital Mortality in COVID-19 Patients. Molecules (2020) 25(23):5725. doi: 10.3390/ molecules 25235725

82. Wang R, He M, Yin W, Liao X, Wang B, Jin X, et al. The Prognostic Nutritional Index Is Associated With Mortality of COVID-19 Patients in Wuhan, China. J Clin Lab Anal (2020) 34(10):e23566. doi: 10.1002/ jcla. 23566

83. Simadibrata DM, Pandhita BAW, Ananta ME, Tango T. Platelet-ToLymphocyte Ratio, a Novel Biomarker to Predict the Severity of COVID19 Patients: A Systematic Review and Meta-Analysis. J Intensive Care Soc (2020), 1751143720969587. doi: 10.1177/1751143720969587

84. Qu R, Ling Y, Zhang Y-H-Z, Wei L-Y, Chen X, Li X-M, et al. Platelet-ToLymphocyte Ratio Is Associated With Prognosis in Patients With Coronavirus Disease-19. J Med Virol (2020) 92(9):1533-41. doi: 10.1002/ jmv. 25767

85. Zheng Y, Huang Z, Ying G, Zhang X, Ye W, Hu Z, et al. Comparative Study of the Lymphocyte Change Between COVID-19 and non-COVID-19 Pneumonia Cases Suggesting Uncontrolled Inflammation Might Not be the Main Reason of Tissue Injury. Medrxiv (2020). doi: 10.1101/ 2020.02.19.20024885

86. Rizo-Téllez SA, Méndez-García LA, Flores-Rebollo C, Alba-Flores F, Alcántara-Suárez R, Manjarrez-Reyna AN, et al. The Neutrophil-ToMonocyte Ratio and Lymphocyte-To-Neutrophil Ratio at Admission Predict In-Hospital Mortality in Mexican Patients With Severe SARSCoV-2 Infection (Covid-19). Microorganisms (2020) 8(10). doi: 10.3390/ microorganisms 8101560

87. Sun D-W, An L, Lv G-Y. Albumin-Fibrinogen Ratio and FibrinogenPrealbumin Ratio as Promising Prognostic Markers for Cancers: An Updated Meta-Analysis. World J Surg Oncol (2020) 18(1):9. doi: 10.1186/ s12957-020-1786-2

88. Yue W, Liu Y, Ding W, Jiang W, Huang J, Zhang J, et al. The Predictive Value of the Prealbumin-to-Fibrinogen Ratio in Patients With Acute Pancreatitis. Int J Clin Pract (2015) 69(10):1121-8. doi: 10.1111/ijcp.12682

89. Shufei Z, Lei S, Jinying Z, Min Y, Jun L, Heyuan D. Prealbumin to Fibrinogen Ratio is Closely Associated With Diabetic Peripheral Neuropathy. Endocr Connections (2020) 9(8):858-63. doi: 10.1530/EC-200316

90. Bi X, Su Z, Yan H, Du J, Wang J, Chen L, et al. Prediction of Severe Illness Due to COVID-19 Based on an Analysis of Initial Fibrinogen to Albumin Ratio and Platelet Count. Platelets (2020) 31(5):674-9. doi: 10.1080/ 09537104.2020.1760230

91. Karakoyun I, Colak A, Turken M, Altin Z, Arslan FD, Iyilikci V, et al. Diagnostic Utility of C-Reactive Protein to Albumin Ratio as an Early Warning Sign in Hospitalized Severe COVID-19 Patients. Int Immunopharmacol (2021) 91:107285. doi: 10.1016/j.intimp.2020.107285

92. Oh J, Kim SH, Park KN, Oh SH, Kim YM, Kim HJ, et al. High-Sensitivity CReactive Protein/Albumin Ratio as a Predictor of in-Hospital Mortality in Older Adults Admitted to the Emergency Department. Clin Exp Emerg Med (2017) 4(1):19-24. doi: 10.15441/ceem.16.158

93. Guo X-L, Zhang Y, Zeng Y-H, Zhao F-Y, Liu W-P, Xiao L, et al. Serum Prealbumin Deserves More Significance in the Early Triage of COVID-19 Patients. Clin Chem Lab Med (CCLM) (2020) 58(10):e209-el1. doi: 10.1515/ cclm-2020-0663

94. Zheng C, Liu S, Feng J, Zhao X. Prognostic Value of Inflammation Biomarkers for Survival of Patients With Neuroblastoma. Cancer Manag Res (2020) 12:2415-25. doi: 10.2147/CMAR.S245622

95. Fang E, Wang X, Feng J, Zhao X. The Prognostic Role of Glasgow Prognostic Score and C-Reactive Protein to Albumin Ratio for Sarcoma: A System Review and Meta-Analysis. Dis Markers (2020) 2020:8736509. doi: 10.1155/ 2020/8736509

96. Onodera T, Goseki N, Kosaki G. Prognostic Nutritional Index in Gastrointestinal Surgery of Malnourished Cancer Patients. Nihon Geka Gakkai Zasshi (1984) 85(9):1001.

97. Jiang N, Deng J-Y, Ding X-W, Ke B, Liu N, Zhang R-P, et al. Prognostic Nutritional Index Predicts Postoperative Complications and Long-Term
Outcomes of Gastric Cancer. World I Gastroenterol (2014) 20(30):10537. doi: $10.3748 /$ wjg.v20.i30.10537

98. Sun K, Chen S, Xu J, Li G, He Y. The Prognostic Significance of the Prognostic Nutritional Index in Cancer: A Systematic Review and MetaAnalysis. J Cancer Res Clin Oncol (2014) 140(9):1537-49. doi: 10.1007/ s00432-014-1714-3

99. Hu B, Yang XR, Xu Y, Sun YF, Sun C, Guo W, et al. Systemic ImmuneInflammation Index Predicts Prognosis of Patients After Curative Resection for Hepatocellular Carcinoma. Clin Cancer Res an Off J Am Assoc Cancer Res (2014) 20(23):6212-22. doi: 10.1158/1078-0432.CCR-14-0442

100. Chiang K-C, Huang S-T, Wu R-C, Huang S-C, Yeh T-S, Chen M-H, et al. Interferon $\alpha$-Inducible Protein 27 is an Oncogene and Highly Expressed in Cholangiocarcinoma Patients With Poor Survival. Cancer Manag Res (2019) 11:1893-905. doi: 10.2147/CMAR.S196485

101. Suomela S, Cao L, Bowcock A, Saarialho-Kere U. Interferon Alpha-Inducible Protein 27 (IFI27) Is Upregulated in Psoriatic Skin and Certain Epithelial Cancers. J Invest Dermatol (2004) 122(3):717-21. doi: 10.1111/j.0022202X.2004.22322.x

102. Wang H, Qiu X, Lin S, Chen X, Wang T, Liao T. Knockdown of IFI27 Inhibits Cell Proliferation and Invasion in Oral Squamous Cell Carcinoma. World J Surg Oncol (2018) 16(1):64. doi: 10.1186/s12957-018-1371-0

103. Hadjadj J, Yatim N, Barnabei L, Corneau A, Boussier J, Smith N, et al. Impaired Type I Interferon Activity and Inflammatory Responses in Severe COVID-19 Patients. Science (2020) 369(6504):718. doi: 10.1126/ science.abc6027

104. Wilk AJ, Rustagi A, Zhao NQ, Roque J, Martínez-Colón GJ, McKechnie JL, et al. A Single-Cell Atlas of the Peripheral Immune Response in Patients With Severe COVID-19. Nat Med (2020) 26(7):1070-6. doi: 10.1038/s41591020-0944-y

105. Zhang N, Zhao YD, Wang XM. CXCL10 an Important Chemokine Associated With Cytokine Storm in COVID-19 Infected Patients. Eur Rev Med Pharmacol Sci (2020) 24(13):7497-505. doi: 10.26355/ eurrev_202007_21922

106. Al Heialy S, Hachim MY, Senok A, Gaudet M, Abou Tayoun A, Hamoudi R, et al. Regulation of Angiotensin- Converting Enzyme 2 in Obesity: Implications for COVID-19. Front Physiol (2020) 11:555039-. doi: 10.3389/fphys.2020.555039

107. Patterson BK, Seethamraju H, Dhody K, Corley MJ, Kazempour K, Lalezari JP, et al. Disruption of the CCL5/RANTES-CCR5 Pathway Restores Immune Homeostasis and Reduces Plasma Viral Load in Critical COVID-19. medRxiv (2020). doi: 10.1101/2020.05.02.20084673 2020.05.02.20084673

108. Zheng H-Y, Xu M, Yang C-X, Tian R-R, Zhang M, Li J-J, et al. Longitudinal Transcriptome Analyses Show Robust T Cell Immunity During Recovery From COVID-19. Signal Transduction Targeted Ther (2020) 5(1):294. doi: 10.1038/s41392-020-00457-4

109. Trouillet-Assant S, Viel S, Gaymard A, Pons S, Richard J-C, Perret M, et al. Type I IFN Immunoprofiling in COVID-19 Patients. J Allergy Clin Immunol (2020) 146(1):206-8.e2. doi: 10.1016/j.jaci.2020.04.029

110. Huang L, Shi Y, Gong B, Jiang L, Liu X, Yang J, et al. Blood Single Cell Immune Profiling Reveals the Interferon-MAPK Pathway Mediated Adaptive Immune Response for COVID-19. Medrxiv (2020). doi: 10.1101/ 2020.03.15.20033472

111. Loganathan T, Ramachandran S, Shankaran P, Nagarajan DJP. Host Transcriptome-Guided Drug Repurposing for COVID-19 Treatment: A Meta-Analysis Based Approach. PeerJ (2020) 8:e9357. doi: 10.7717/ peer. 9357

112. Shaath H, Vishnubalaji R, Elkord E, Alajez NM. Single-Cell Transcriptome Analysis Highlights a Role for Neutrophils and Inflammatory Macrophages in the Pathogenesis of Severe COVID-19. Cells (2020) 9(11). doi: 10.3390/ cells 9112374

113. Mick E, Kamm J, Pisco AO, Ratnasiri K, Babik JM, Castañeda CS, et al. Upper Airway Gene Expression Differentiates COVID-19 From Other Acute Respiratory Illnesses and Reveals Suppression of Innate Immune Responses by SARS-CoV-2. Nat Commun (2020) 11(1):5854. doi: 10.1038/s41467-02019587-y.

114. Alsamman AM, Zayed H. The Transcriptomic Profiling of COVID-19 Compared to SARS, MERS, Ebola, and H1N1. PLoS One (2020) 15(12): e0243270. doi: 10.1371/journal.pone.0243270 
115. Hammadi AA, Al Jubouri AM, Ahmed G, Hayyawi AH, Kareem K, Gorial FI, et al. A New Hematological Prognostic Index For Covid-19 Severity. Medrxiv (2021). doi: 10.1101/2021.02.11.21251285

116. Lidoriki I, Frountzas M, Schizas D. Could Nutritional and Functional Status Serve as Prognostic Factors for COVID-19 in the Elderly? Med Hypotheses (2020) 144:109946. doi: 10.1016/j.mehy.2020.109946

117. SeyedAlinaghi S, Mirzapour P, Dadras O, Pashaei Z, Karimi A, MohsseniPour M, et al. Characterization of SARS-CoV-2 Different Variants and Related Morbidity and Mortality: A Systematic Review. Eur $J$ Med Res (2021) 26(1):51. doi: 10.1186/s40001-021-00524-8

Conflict of Interest: The authors declare that the research was conducted in the absence of any commercial or financial relationships that could be construed as a potential conflict of interest.
Publisher's Note: All claims expressed in this article are solely those of the authors and do not necessarily represent those of their affiliated organizations, or those of the publisher, the editors and the reviewers. Any product that may be evaluated in this article, or claim that may be made by its manufacturer, is not guaranteed or endorsed by the publisher.

Copyright (c) 2021 Karimi, Shobeiri, Kulasinghe and Rezaei. This is an open-access article distributed under the terms of the Creative Commons Attribution License (CC BY). The use, distribution or reproduction in other forums is permitted, provided the original author(s) and the copyright owner(s) are credited and that the original publication in this journal is cited, in accordance with accepted academic practice. No use, distribution or reproduction is permitted which does not comply with these terms. 